\title{
Des lois de l'induction aux théorèmes de Thévenin et de Norton
}

\author{
Maria Timofeeva ${ }^{1}$, Gilles Allègre ${ }^{2}$, Stéphane Flament ${ }^{2}$ et Didier Robbes ${ }^{1}$ \\ maria.timofeeva@inphynix.com \\ Adresses : (1) Inphynix, Plug N'Work, Bâtiment D, 2 rue jean Perrin, 14460, COLOMBELLES
}

(2) GREYC - UMR 6072, 6 Bd Mal Juin, 14050 CAEN Cedex

\begin{abstract}
RESUME : Les lois de Lenz et de l'auto-induction sont étudiées pour une boucle conductrice circulaire monotour, dans une gamme de fréquence de $1 \mathrm{~Hz}$ à $1 \mathrm{MHz}$. La fem en circuit ouvert et le courant de court circuit permettent au final d'obtenir les schémas équivalents de Thévenin et de Norton de la spire sous influence magnétique. La mesure de la tension en circuit ouvert met en œuvre un amplificateur (de tension) large bande à bas bruit. La mesure du courant de court circuit est réalisée d'une part avec un amplificateur à transimpédance lui aussi large bande et à bas bruit et d'autre part en mesurant l'induction magnétique locale au voisinage de la spire fermée sur elle même. Cette dernière est comparée aux calculs analytiques et numériques. La comparaison des deux mesures du courant de court circuit permet de remonter à l'impédance d'entrée de l'amplificateur à transimpédance. Cette méthode peut être utilement étendue pour des circuits couplés (transformateur élémentaire, transformateur de flux) et pour l'étude de l'effet de peau.
\end{abstract}

Mots clés : loi de Lenz, loi de l'auto-induction, théorème de Thévenin, théorème de Norton.

\section{INTRODUCTION}

Le passage des représentations du physicien à celles de l'électronicien pose parfois des difficultés pédagogiques; il est le point de départ des activités présentées dans cet article. Les années 1 et 2 des licences des domaines de la physique et des sciences de l'ingénieur, introduisent les notions fondamentales de l'électromagnétisme et de l'électro-cinétique. Sur ces bases, l'enseignant d'EEA tire l'essence des outils adaptés à la manipulation des grandeurs électriques, réduites à la paire tension - courant, (charge - flux dans une moindre mesure), autour de dipôles et de quadripôles. Ces variables se relient via des théorèmes fondamentaux, dont ceux de Thevenin et Norton. La médiation entre les deux types d'enseignants, physicien - enseignant EEA, n'est pas favorisée par l'organisation actuelle des enseignements. Pour les étudiants, le passage d'un mode de représentation à l'autre peut s'avérer délicat. Nous proposons une série d'expériences visant à aider ce passage. Un circuit électrique "académique" constitué d'une spire filaire monotour en cuivre (fils de Litz), est sous influence magnétique large bande produite par une paire de bobines circulaires en position de Helmoltz. La force électromotrice résultant de la loi de Lenz, puis le courant circulant dans la boucle résultant des lois de l'induction, sont mesurés. Les caractéristiques des générateurs équivalents de Thevenin et de Norton de la spire sous influence magnétique sont directement déduites, pour des fréquences comprises entre quelques $\mathrm{Hz}$ et $1 \mathrm{MHz}$.

\section{PRESENTATION DE L'EXPERIMENTATION - SIMULATIONS}

\subsection{Description du banc d'expériences}

Afin de ne pas dérouter les apprenants, le cadre de cette expérimentation est celui d'un cas d'école classique : le circuit filiforme circulaire (spire) dans un champ uniforme variable dans le temps. L'axe de la spire est colinéaire au champ. Cette configuration est axi-symétrique ce qui permet une formulation à deux dimensions.

La génération du champ uniforme est assuré par deux bobines circulaires de $20 \mathrm{~cm}$ de diamètre en position de Helmoltz ( 25 tours chacune en fils de Litz de 30 brins en cuivre de $50 \mu \mathrm{m}$ de diamètre). La spire d'étude, circulaire monotour de $8 \mathrm{~cm}$ de diamètre est placée proche de son plan de symétrie (fig1). Celle ci est constitué de trois fils de Litz branchés en parallèle et présente deux connectiques différentes, adaptées à une mesure en circuit ouvert (avec un circuit de garde) ou en circuit fermé (avec le point diamétralement opposé à l'arrivée du câble (point milieu) qui est utilisé comme potentiel de référence).

Pour une utilisation à des fréquences allant au mégahertz il est nécessaire prendre en compte l'effet de peau. L'épaisseur de peau $\delta=\sqrt{\frac{2}{\mu_{0} \sigma \omega}}$ est de $66 \mu \mathrm{m}$ à $1 \mathrm{MHz}$ pour du cuivre. Avec le fil de Litz utilisé, l'effet n'apparaît donc qu'au delà du domaine étudié.

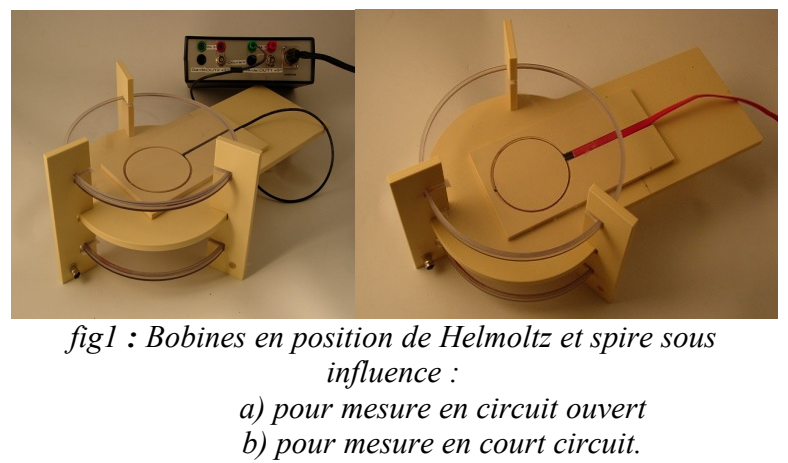

2.2 Etude du champ créé et des grandeurs intégrales associées

Dans l'hypothèse où les câbles de connexion (torsadés et blindés) permettent de rendre négligeable l'effet des courants y circulant, ou bien en réalisant l'étude dans une zone bien choisie de l'espace, on peut considérer que le champ est engendré uniquement par les courants des circuits filiformes circulaires (bobines de Helmoltz et bobine sous influence). Ce montage apparaît donc 
comme la superposition de trois montages élémentaires similaires ; pour chacun d'entre eux, le problème posé est celui du champ créé par une spire circulaire coaxiales. Les supports théoriques de base [1] qui autorisent le calcul de l'induction magnétique en tout point sont l'intégration de la loi de Biot et Savart $\vec{B}=\frac{\mu_{0} I}{4 \pi} \int d \vec{I} \times \frac{\vec{r}}{r^{3}}$ ou l'utilisation de l'expression $\mathrm{du}$ potentiel vecteur (avec $a$ rayon de la spire) $A_{\varphi}=\frac{\mu_{0} I}{4 \pi} \int_{C} \frac{d l_{y}}{r}=\frac{\mu_{0} I}{2 \pi} \int_{0}^{\pi} \frac{a \cos \varphi \cdot d \varphi}{\sqrt{a^{2}+\rho^{2}+z^{2}-2 a \rho \cos \varphi}}$ associée à l'équation de définition $\vec{B}=\overrightarrow{r o t} \vec{A}$. S'il est nécessaire d'utiliser le formalisme des intégrales elliptiques de Legendre pour avoir une expression analytique de $\vec{B}$, l'approche pédagogique simple qui consiste à utiliser un tableur pour estimer numériquement le résultat théorique est préféré [2]. Il constitue une bonne approche des méthodes utilisées dans les outils de simulation.

Déjà abordé dans d'autres travaux pratiques [2], l'aspect local/intégral des équations est ici simplement réinvesti à travers l'utilisation d'un logiciel de simulation numérique par élément finis [3] pour visualiser la topographie du champ (fig2). La figure, pour sa moitié haute, correspond à l'étude de la spire sous influence en circuit ouvert et pour sa moitié basse, à l'étude en court circuit. Les inclusions présentent un zoom sur le voisinage de la section de la spire. La simulation est par essence même à symétrie axiale et il faut insister ici sur la géométrie choisie pour la manipulation (bobine en position de Helmoltz) qui autorise une manipulation peu sensible à la position de la spire d'étude.

Les deux configurations circuit ouvert et court circuit sont simulées en paramétrant les propriétés de la spire. Le logiciel la considère soit comme un circuit source de courant nul (circuit ouvert fig2 haut) soit comme du matériau "passif" (c.a.d. en court circuit fig2 bas) dans lequel se développe les courants induits. Il apparaît nettement sur les zooms l'effet local du courant induit par le courant de court circuit $\mathrm{I}_{\mathrm{cc}}$.

La possibilité d'accéder à Icc dans le logiciel autorise ensuite à ne regarder l'effet que de ce seul courant et de calculer le champ qu'il produit. En le sommant avec le champ généré par les bobines de Helmoltz quand la spire est en circuit ouvert, on peut vérifier le théorème de superposition au niveau physique. La superposition correspond en effet au champ total calculé par le logiciel lorsque les bobines de Helmoltz induisent un courant dans la spire en court circuit.

Enfin, la possibilité de choisir le type de fils de cuivre pour la spire sous influence permet la mise en évidence de l'effet de peau. Dans le cas de la manipulation (fils de Litz), la simulation confirme que la densité de courant dans la spire reste bien indépendante de la fréquence, ce qui n'est plus le cas lorsque le choix est fait de prendre du cuivre massif.

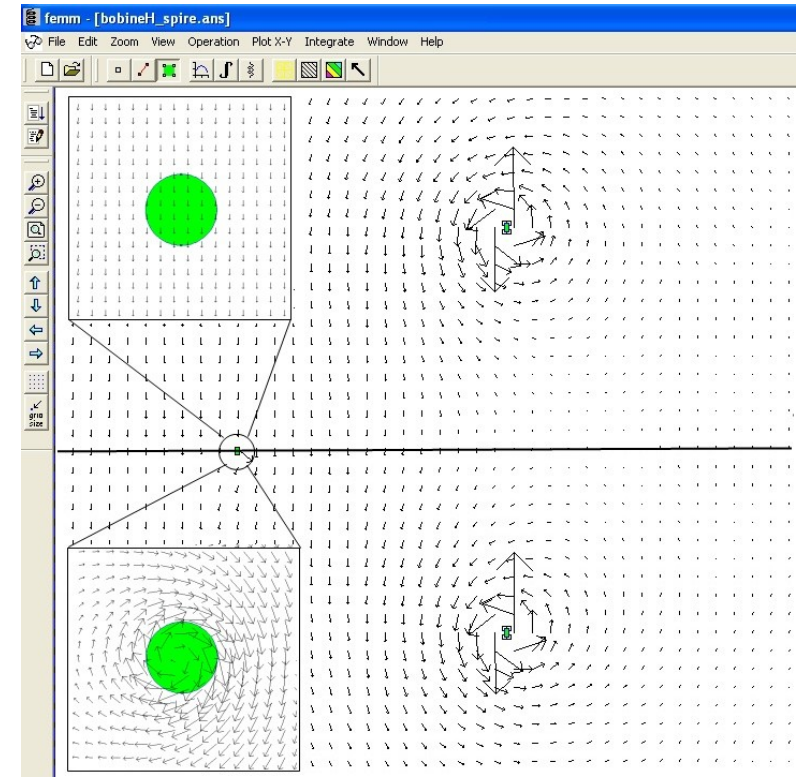

Topographie des lignes de champ. fig2 haut) : spire en circuit ouvert, fig2 bas) : spire en court circuit.

Les zooms (encadrés) détaillent le champ près de la spire et l'effet du courant induit pour la spire en court circuit.

D'un point de vue électro-cinétique, les grandeurs développées dans le circuit sont obtenues d'une part grâce aux équations de Maxwell et d'autre part aux caractéristiques du matériau constituant le circuit.

L'expression du champ électrique est obtenue par son équation de définition $\vec{E}=-\overrightarrow{\operatorname{gra} \vec{d} V}-\frac{\partial \vec{A}}{\partial t}$ dont la circulation le long de la spire donne :

$$
\int_{A}^{B} \vec{E} \cdot \overrightarrow{d r}=-\left(V_{B}-V_{A}\right)-\frac{\partial}{\partial t} \int_{A}^{B} \vec{A} \cdot \overrightarrow{d r}(1) .
$$

Coté matériau, la forme locale de la loi d'Ohm $\vec{J}=\sigma \vec{E}$ permet le calcul du premier membre de (1) :

$$
\int_{A}^{B} \vec{E} \cdot \overrightarrow{d r}=\int_{A}^{B} \frac{1}{\sigma} \vec{j} \overrightarrow{d r}=\int_{A}^{B} \frac{i \cdot d r}{\sigma S}=R_{A B} \cdot i
$$

Pour le deuxième membre, deux cas se présentent en pratique :

- le circuit est ouvert et on calcule la fém à vide avec $\mathrm{A}$ qui tend vers B sans contact électrique ;

- le circuit est fermé et on calcule un courant induit de court circuit $\mathrm{I}_{\mathrm{cc}}$ avec A qui est confondu avec B.

Dans les deux cas le terme de circulation du potentiel vecteur dans le second membre de (1) se calcule sur un contour fermé. Avec l'équation de définition de $\vec{B}$ et l'équation de conservation de Maxwell-Thomson on définit le flux de $\mathrm{B}$ qui ne dépend plus que de $\mathrm{t}$ :

$$
\Phi(t)=\int_{S} B \cdot n d S=\int_{S} \overrightarrow{\operatorname{rot}} A \cdot n d S=\oint_{C} A \cdot d r
$$

En circuit ouvert, $\mathrm{i}=0$ et le champ B est uniquement du aux bobines de Helmoltz d'où $\Phi(t)=\Phi_{\text {Helm }}(t)$. Avec (2) et (3) et en posant $e(t)=V_{B}-V_{A}$ à vide, (1) devient : 


$$
e(t)=-\frac{d \Phi_{H e l m}(t)}{d t}
$$

ou loi de Lenz-Faraday. On notera que e(t) aux bornes de la bobine est en convention générateur compte tenu de l'orientation de la circulation.

En circuit fermé, $V_{A}=V_{B}$ et le courant induit créé un flux propre $\Phi_{\text {propre }}(t)=L i$ D'où

$$
\begin{gathered}
\Phi(t)=\Phi_{\text {Helm }}(t)+\Phi_{\text {propre }}(t) \quad \text { et } \quad \text { (1) devient } \\
R \cdot i=-\frac{d \Phi}{d t}=-\frac{d \Phi_{\text {Helm }}}{d t}-L \frac{d i}{d t} \text { soit : } \\
e(t)=L \frac{d i}{d t}+R \cdot i \text { (5) }
\end{gathered}
$$

avec e(t) définit par (4).

Il apparaît ainsi que la tension à vide donne l'image de la dérivée du flux alors que le courant de court circuit donne l'image du flux lui même pour peu que la fréquence d'étude soit au dessus de la fréquence de coupure intrinsèque de la spire : $f>>\frac{R}{2 \pi L}$.

\subsection{Modèles de Thévenin et de Norton}

Cette approche physique est à confronter maintenant à celle électronicienne du dipôle électrique que constitue la spire. Fondée sur la linéarité mathématique de toutes les équations mise en jeu, les deux approches duales de Thévenin ou de Norton permettent la modélisation de la spire par deux générateurs réels (de tension ou de courant) du même nom [4]. Les valeurs de ces générateurs sont obtenus par un couple de mesure en circuit ouvert (donnant $\mathrm{E}_{\mathrm{Th}}$ ) et en court circuit (donnant $\mathrm{I}_{\mathrm{N}}$ ). La détermination de l'impédance ou admittance de source se fait par le rapport des grandeurs précédentes ou par une mesure directe.

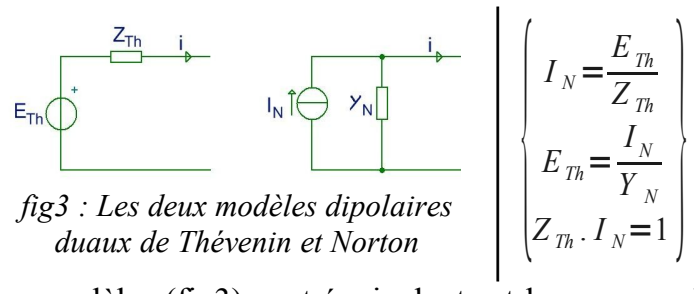

Les modèles (fig3) sont équivalents et leurs paramètres sont donnés par le système d'équation (6) dont la symétrie met en exergue la dualité des approches.

Avec (4), la tension de Thévenin s'exprime théoriquement par $E_{T h}=-j \omega \Phi$ où, pour simplifier l'écriture, on notera pour la suite $\Phi(t)=\Phi_{\text {Helm }}(t)$. Avec (5) le courant de Norton est donné par $I_{N}=\frac{-j \omega \Phi}{R+j L \omega}=-\frac{\Phi}{L} \frac{1}{1-j \frac{R}{L \omega}}$. Ceci débouche évidemment pour l'impédance de source sur: $Z=R+j \omega L$.

La simulation sous FEMM donne accès aux grandeurs complexes électriques donc à $\mathrm{E}_{\mathrm{Th}}, \mathrm{I}_{\mathrm{N}} \mathrm{R}$ et $\mathrm{L}$. Le relevé pour plusieurs fréquences de ces grandeurs permet de confronter la simulation aux expressions théoriques précédentes de $E_{T h}$ et $I_{N}, R$ et $L$ étant donnés directement.

Avec un courant dans les bobines de Helmoltz de $10 \mathrm{~mA}$, la simulation donne comme valeur :

$\mathrm{B}_{\text {Helm }}=2 \mu \mathrm{T}, \quad \Phi_{\text {Helm }}=10 \mathrm{nWb}, \quad \mathrm{E}_{\mathrm{Th}}=-\mathrm{j} .6,28 \mathrm{e}^{-8} . \mathrm{f}$, $\mathrm{R}=24,4 \mathrm{~m} \Omega$ et $\mathrm{L}=2,6 \mathrm{e}^{-7} \mathrm{H}$. Concernant $\mathrm{I}_{\mathrm{N}}$, on vérifie bien que la dépendance en fréquence correspond à l'expression théorique.

\subsection{L'électronique de mesure}

Pour confronter théorie et simulation à la mesure, l'accès expérimental au champ se fait grâce à une sonde de champ large bande [5]. L'accès expérimental aux grandeurs électriques, tension puis courant, se fait grâce à une électronique adaptée. Cette partie donne les caractéristiques des amplificateurs large bande et faible bruit réalisés. Elle sera plus détaillée pour la partie transimpédance dont les caractéristiques sont moins classiques.

L'utilisation d'un simulateur électronique [6] permet aux étudiants de visualiser les notions liées aux systèmes contre réactionnés en terme de bande passante, d'impédances,...

\subsubsection{Caractéristique de la source}

La spire est un dipôle dont le modèle, que ce soit dans l'approche de Thévenin ou de Norton, comporte une impédance qui peut se déterminer électriquement (hors influence) comme cela sera réalisé en pratique.

Par la simulation il est possible d'appréhender la dynamique des signaux; $\left|\mathrm{E}_{\mathrm{th}}\right|$ varie entre $63 \mathrm{nV}$ et $63 \mathrm{mV}$ et $\left|\mathrm{I}_{\mathrm{N}}\right|$ varie entre $2,6 \mu \mathrm{A}$ et $31 \mathrm{~mA}$ dans la gamme de fréquence $1 \mathrm{~Hz}$ à $1 \mathrm{MHz}$.

Ainsi, pour la mesure en circuit ouvert, il faut accéder à une tension à vide de faible niveau à faible impédance de source. Le cahier des charges de l'électronique est alors celui d'un amplificateur de tension classique faible bruit (large bande) à très forte impédance d'entrée (devant celle de la source).

Pour la mesure en court circuit, il faut accéder à un courant à faible impédance de source. Le cahier des charges de l'électronique est alors celui d'un amplificateur à transimpédance (large bande) à très faible impédance d'entrée (devant celle déjà faible de la source).

Etant donné la dynamique attendue, le montage est conçu pour une avoir une transimpédance $\mathrm{z}_{\mathrm{m}}=2 \mathrm{~V} / \mathrm{A}$ et présenter à la source une entrée à très basse impédance de l'ordre de quelques $\mu \Omega$ en continu.

\subsubsection{L'amplification de tension}

Dans l'approche de Thévenin, la mesure de la tension en circuit ouvert de faible niveau sous faible impédance de source est très classiquement réalisée par un 
amplificateur de tension large bande à grande impédance d'entrée et à faible bruit. La structure est classique à base d'un amplificateur linéaire intégré (ALI) différentiel à gain variable. Seule une structure de garde de mode commun est utilisée en plus pour se prémunir des couplages capacitifs entre les conducteurs du câble et la terre via le blindage (fig4). Le mode commun est obtenu par la réalisation d'un point milieu sur la résistance externe de réglage du gain.

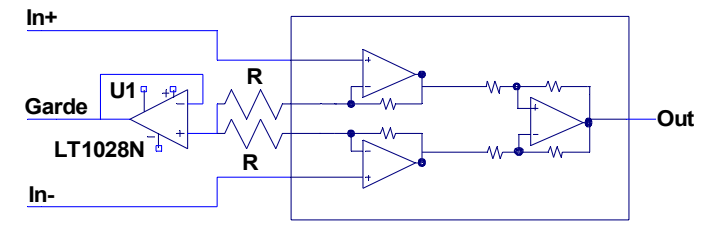

fig4 : Schéma de l'amplificateur de tension avec garde.

La structure non inverseuse présente de part la contre réaction une bande passante supérieure à $1 \mathrm{MHz}$ lorsque le gain reste inférieur à $40 \mathrm{~dB}$ et une impédance d'entrée qui est celle de l'amplificateur multiplié par le gain de boucle, donc excessivement grande. La simulation confirme les résultats théoriques.

On remarque surtout à l'occasion de ce montage la technique de garde qui consiste à asservir la tension du blindage à celle de l'âme "effaçant" ainsi la capacité.

\subsubsection{L'amplification à transimpédance}

Dans l'approche de Norton, la mesure du courant de court circuit (virtuel) de faible niveau sous faible impédance de source est réalisée par un amplificateur différentiel à transimpédance lui aussi large bande à très faible impédance d'entrée avec branchement en 5 points.

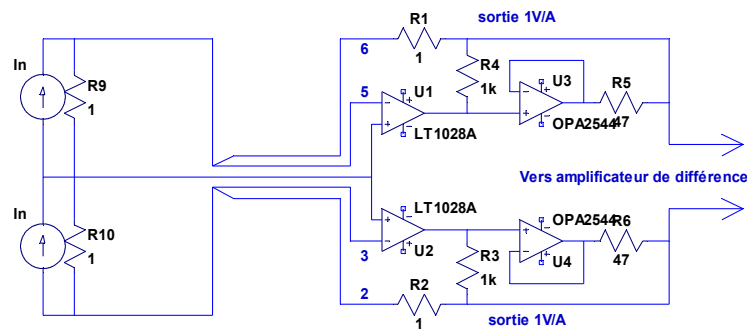

fig5 : Schéma de l'amplificateur à transimpédance

L'analyse du schéma (fig5) montre une conversion courant tension à base d'ALI attaqués sur l'entrée inverseuse avec une contre réaction en courant. Cette structure permet d'obtenir une grande bande passante ainsi qu'une faible impédance d'entrée (voir équations ci-après).Un OPA2544 sert de buffer de courant pour atteindre plusieurs centaines de milliampère. La résistance de réaction est de $48 \Omega$. La tension de sortie est élaborée par un amplificateur de différence, soit avec une transimpédance globale de 2V/A.

La structure différentielle est utilisée principalement pour éliminer les composantes de mode commun induites par les flux dans les boucles résiduelles des câbles. La spire est donc réalisée avec un point milieu qui dédouble le flux en deux demi-flux différentiels actifs. Les deux étages d'entrée de la structure différentielle sont construits autour de composants faible bruit (LT1028).

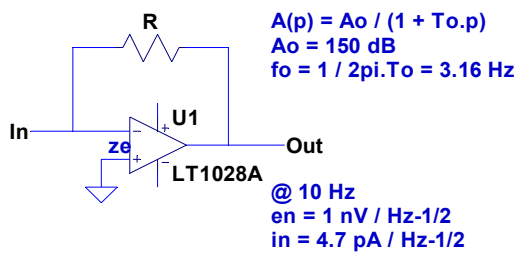

fig6 : Schéma de base d'un l'amplificateur à transimpédance

Le montage de base utilisé ici pour la conversion courant tension (fig6) est simple. Sa structure est classique mais son utilisation et la pratique de ces équations restent peu usités à l'instar de "l'approche courant". Nous nous y attarderons donc. Nous avons $V_{\text {out }}=A(p) \cdot\left(V_{+}-V_{-}\right) \quad$ où $\quad V_{+}=0 \quad$ et $I_{\text {in }}=\frac{V_{-}}{z_{e}}+\frac{\left(V_{-}-V_{\text {out }}\right)}{R}$ qui donnent le transfert en substituant $\mathrm{V}-$ :

$$
\frac{V_{\text {out }}}{I_{I n}}=-\frac{R}{1+\frac{1}{A(p)}\left(1+\frac{R}{z_{e}}\right)} \approx-R \frac{A(p)}{1+A(p)}
$$

ainsi que l'impédance d'entrée en substituant $\mathrm{V}_{\text {out }}$ :

$$
z_{e}=\frac{V_{-}}{I_{I n}}=\frac{-1}{A(p)} \frac{V_{\text {Out }}}{I_{I n}} \approx R \frac{1}{1+A(p)}
$$

Ces expressions font intervenir les termes habituels des systèmes bouclés à retour unitaire. Une étude asymptotique de (7) et (8) montre que la bande passante est égale à la fréquence de transition de l'ALI et que $Z_{\mathrm{e}}$ est résistive de très faible valeur $\frac{R}{A_{0}}$ jusqu'à $\mathrm{f}_{\mathrm{o}}$ puis est selfique au delà avec $L=\frac{R \tau_{0}}{A_{0}}$. Pour fixer les idées l'impédance d'entrée devient égale à $\mathrm{R}$ à la fréquence de transition de l'ALI.

La fonction de transfert de l'amplificateur réalisé a été simulée. Elle est plate jusqu'à $1 \mathrm{MHz}$. Son impédance d'entrée différentielle $\left(2 . Z_{\mathrm{e}}\right)$ est la représentation la plus intéressante et est comparée à l'impédance de source $\mathrm{Z}$ (fig7).

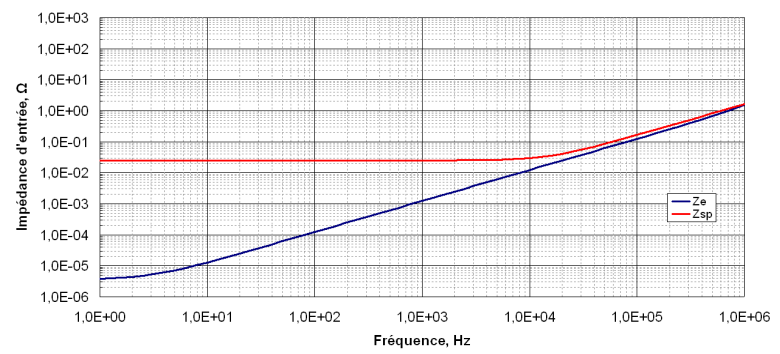

fig7 : Relevé de l'impédance d'entrée différentielle en fonction de la fréquence (simulation) et tracé de l'impédance de source théorique. 


\section{RÉSULTATS EXPÉRIMENTAUX}

\subsection{Mesure du champ}

L'accès expérimental au champ se fait grâce à une "sonde M" [5]. Un relevé est réalisé sur un rayon $(\mathrm{z}=\mathrm{cste})$ et peut être confronté à la simulation qui permet l'extraction du profil correspondant. Cette méthode est aussi utilisée pour la mesure du courant d'une spire en vrai court circuit (réel) et sera illustrée à cette occasion (fig9).

\subsection{Validation des mesures avec l'amplificateur à transimpédance}

Compte tenu du modèle de la source de signal il est nécessaire de vérifier la valeur réelle de l'impédance d'entrée de l'amplificateur à transimpédance. En effet celle-ci est susceptible de ne pas très rester faible devant l'impédance déjà faible de la source. La mesure du courant de court circuit virtuel par amplification à transimpédance est donc qualifiée par comparaison au courant de court circuit réel.

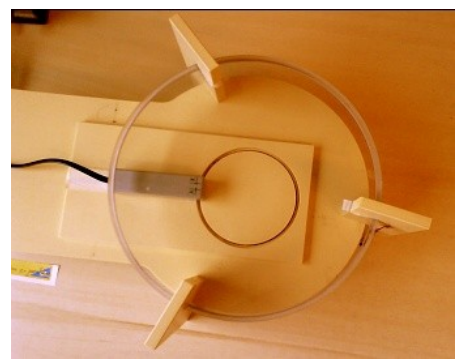

fig8 : Dispositif pour l'étude du courant de court circuit réel (le câble est celui de la sonde de champ)

Pour accéder à ce courant une mesure magnétique locale de l'induction (fig8) dans une direction orthogonale au champ des bobines de Helmoltz et au voisinage de la spire en court circuit réel permet d'y remonter.

Sur la figure 9 sont comparés un relevé expérimental du champ réalisé sur un rayon $(\mathrm{z}=\mathrm{cste})$, celui attendu en simulation et celui de la théorie.
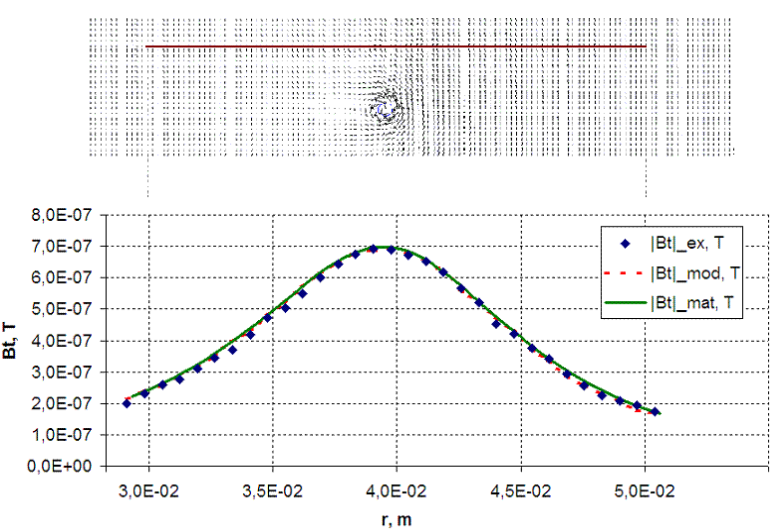

fig9 : Comparaison des tracés théorique (mat), simulé (mod) et expérimental (ex) le long du profil rectiligne

Cela permet de valider la procédure expérimentale car, si la composante radiale du champ créé par les bobines de Helmoltz est pratiquement nulle, la mesure reste très sensible à la proximité du bord et surtout à toute non perpendicularité stricte de la sonde de mesure.

Pour la mesure du courant de court circuit virtuel, le relevé en champ est effectué avec la spire reliée à l'amplificateur à transimpédance et sur un rayon diamétralement opposé au câble de liaison (pour s'affranchir de toute éventuelle influence de celui-ci). Le rapport entre les valeurs vectorielles (complexes) expérimentales et les valeurs simulées donne accès à la valeur de l'atténuation du pont diviseur de courant du générateur de Norton entre l'admittance de source et l'admittance d'entrée. On en déduit cette dernière grâce à $i_{c c \text {-vituel }}=I_{n} \cdot \frac{Y_{e}}{\left(Y_{n}+Y_{e}\right)}$. Les mesures confirment qu'en dessous de quelques $\mathrm{kHz}$ l'impédance d'entrée est difficile à estimer car elle est nettement plus faible que celle de la spire et l'amplificateur fonctionne "idéalement". $\mathrm{Au}$ dessus, son impédance d'entrée devient mesurable car plus proche de celle de la spire et l'amplificateur fonctionne non idéalement et sa sortie suppose une correction.

Cette limite de fonctionnement est mise à profit pour bien insister auprès des étudiants sur les nombreux aller-retour constructifs entre théorie, simulation, expérimentation, limitation, amélioration et compensation...

\subsection{Mesures et obtention des schémas équivalents}

\subsubsection{Mesure directe de l'impédance de la spire}

Une mesure de l'impédance de la spire seule en fonction de la fréquence permet de remonter, par ajustement à un modèle dipolaire Rs, Ls série. Le relevé de la fonction de transfert d'un pont diviseur $\left(1 \mathrm{k} \Omega, \mathrm{Z}_{\mathrm{s}}\right)$ donne après identification :

$$
\begin{gathered}
Z_{\mathrm{s}}=\left(25+\mathrm{j} 3,45 \mathrm{e}^{-3} . \mathrm{f}\right) \mathrm{m} \Omega, \text { soit }: \\
\mathrm{R}=25 \mathrm{~m} \Omega, \mathrm{L}=5,5 \mathrm{e}-{ }^{7} \mathrm{H} .
\end{gathered}
$$

\subsubsection{Relevé de la fem à vide : générateur idéal de Thévenin}

La figure 10 montre la comparaison entre les données relevées et l'ajustement correspondant au modèle théorique.

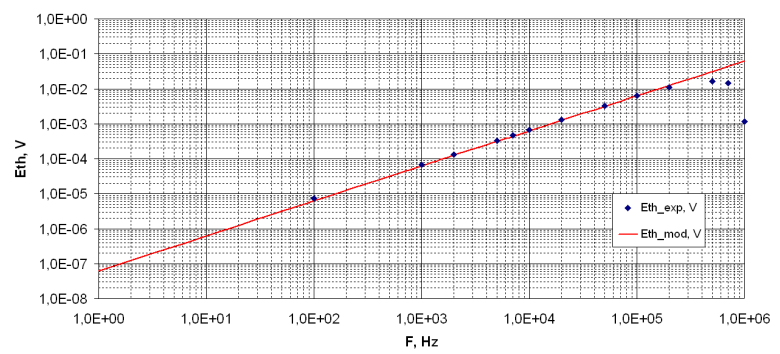

fig10 : Comparaison des tracés expérimentaux et théoriques pour la loi de Lenz, "circuit ouvert"

On observe la parfaite adéquation sur la plage $100 \mathrm{~Hz}-$ $200 \mathrm{kHz}$ qui est limitée en basse fréquence par le bruit 
de l'amplificateur et en haute fréquence par la résonance des bobines de Helmoltz.

Le modèle de la source après identification donne $E_{T h}=-j 6,28 e^{-8}$.f qui correspond idéalement à la théorie. Une compensation de l'affaiblissement dû aux bobines montrerait l'extension de la plage de validité.

\subsubsection{Relevé du courant de court circuit virtuel : générateur idéal de Norton}

La figure 11 montre la comparaison entre les données relevées et l'ajustement du modèle théorique. Les mêmes remarques qu'en 3.3.1 peuvent être faites.

La coupure indique le passage du générateur en mode "fluxmètre" pour $\mathrm{f}=7 \mathrm{kHz}$ avec une valeur asymptotique de 14,3 mA. Une compensation est ici aussi possible.

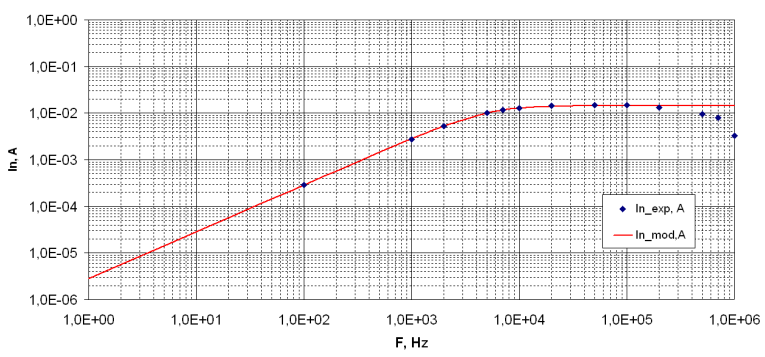

fig11 : Comparaison des tracés expérimentaux et théoriques pour le courant de court circuit virtuel

Le modèle de la source après identification donne $I_{N}=-j 6,28 e^{-4} . f /\left(22 e^{-3}+j 7 e^{-7} \omega f\right)$ ce qui correspond à une résistance de maille de $22 \mathrm{~m} \Omega$ et une inductance de $7 \mathrm{e}^{-7} \mathrm{H}$. Compte tenue de la mesure directe de l'impédance de la spire (3.3.1) il est possible de remonter à l'impédance différentielle de l'amplificateur : sa résistance n'est pas raisonnablement mesurable (elle serait négative) et son inductance est $1,5^{\mathrm{e}-7} \mathrm{H}$ soit environ $10 \mathrm{~m} \Omega$ à $10 \mathrm{kHz}$ ce qui correspond à la simulation.

\subsubsection{Comparaison des résultats}

Il est ainsi possible de confronter les résultats des mesures entre les méthodes (théorie, simulation et expérimentation). Eth correspond idéalement à la théorie. Par contre le générateur de Norton et donc l'impédance de source présentent un écart à la théorie ou à la simulation : on obtient pour $\mathrm{R}_{\mathrm{N}}=22$ à $25 \mathrm{~m} \Omega$ et $\mathrm{L}_{\mathrm{N}} \sim 5,5 \mathrm{e}^{-7} \mathrm{H}$. Ces résultats sont à comparer aux valeurs théoriques attendues $(24,4 \mathrm{~m} \Omega$ et $260 \mathrm{nH})$.

La valeur de $\mathrm{R}$ correspond bien. Celle de L présente une différence de quelques centaines de $\mathrm{nH}$, imputable à l'inductance résiduelle du circuit de mesure actuellement non optimisé pour de si petites valeurs.

Ainsi la spire se comporte-t-elle bien comme son modèle $Z_{T h}=\frac{1}{Y_{N}}=R+j \omega L$.

\section{DISCUSSION ET APPROFONDISSEMENTS}

Ce travail présente également des intérêts pour le niveau L3 ou M1 en électronique du fait des aspects large bande, faible bruit et transimpédance.

De plus des expériences complémentaires à boucles circulaires couplées sont proposées, utiles à l'approche du modèle quadrupolaire du transformateur quasi idéal et du transformateur de flux. Ces expériences de détermination de paramètres $[\mathrm{z}]$ ou $[\mathrm{y}]$ demandent en effets des mesures en circuit ouvert ou en court circuit.

Il est possible aussi d'imaginer des expérimentations de mise en évidence de l'effet de peau, ce qui est peu (ou pas) fait de façon simple. Par exemple, un cylindre creux conducteur placé en alignement dans le champ serait le siège de courants induits dont la répartition spatiale et la phase deviendrait non uniforme lorsque l'épaisseur de peau avoisinerait l'épaisseur du cylindre. Avec en son centre d'une spire de mesure de flux il serait possible de visualiser et de quantifier le phénomène.

\section{Remerciements :}

Nous tenons à remercier les acteurs des dispositifs institutionnels pour leur soutien: l'Université de Caen Basse Normandie (UCBN), le Conseil Régional de Basse Normandie (CRBN), Normandie Incubation, OSEO-Anvar, l'ANRT, SYNERGIA. Une attention particulière va à M. Jean François Le Bourhis, IA-IPR de Sciences Physiques pour son soutien constant.

\section{Bibliographie}

[1] E.Durand, Magnétostatique, Masson, 1968.

[2] Didier Robbes, Thierry Salley, Fréderic Dijoux, Basile Dufay, Gilles Allègre, Maria Timofeeva, S. Morteau, "Autour des équations de Maxwell", accepté à CETSIS 2010.

[3] David Meeker, "Finite Element Method Magnetics, FEMM", Windows finite element solver for $2 D$ and axisymmetric magnetic, electrostatic, heat flow, and current flow problems with graphical pre- and postprocessors.

[4] J. Blot, "Electronique linéaire, cours avec exercices et travaux pratiques", DUNOD, Paris, 1993, ISBN 210 0011332

[5] Société Inphynix, "Magnétomètre sonde M et conditionneur", http://www.inphvnix.com/details produits.php?p id =5

[6] Linear Technology, "High performance Spice III simulator, schematic capture and waveform viewer", http://www.linear.com/designtools/softwarel. 